Laboratories specialising in low temperatures offer natural meeting points for research and technical development, where progress today lies at the crossroads between frustration and disorder and their quantum-level coherence and dynamics.

\title{
Low-Temperature Physics Today
}

Low temperatures have long been common in condensed-matter physics, and more recently in atomic physics (they have always been so in theory, of course, with the basic concepts of the ground state and elementary excitations). Some laboratories - the CRTBT among others - are squarely dubbed centres for low-temperature research. A concentration of powerful cooling facilities and material means allows us to tackle a variety of problems, and turn to new ones relatively rapidly. Rather than list the various fields a laboratory such as ours can tackle, we shall adopt a more generic point of view. The various essential "functions", and the relevant interfaces with other fields, of a centre for low-temperature research will by presented vertically, so to speak, without attempting to be exhaustive.

The founding masterpiece of low-temperature physics dates back to 1908 with the liquefaction, at Leiden, of ${ }^{4} \mathrm{He}$. This made available temperatures $T$ of $4 \mathrm{~K}$ and opened the way, three years later and again in Kamerlingh Onnes's group, to the discovery of superconductivity. By pumping on the liquid, the minimum temperature reached is $1.1 \mathrm{~K}$ for ${ }^{4} \mathrm{He}$ and $0.3 \mathrm{~K}$ for ${ }^{3} \mathrm{He}$. Lower temperatures were eventually obtained by adiabatic demagnetization, but steady-state operation had to wait for ${ }^{3} \mathrm{He}-4 \mathrm{He}$ dilution refrigerators which progressed down to the $\mathrm{mK}$ range in the early 1970 's. These cryostats are based on the Fermi liquid properties of ${ }^{3} \mathrm{He}$ at very low temperatures. In fact, in contrast to the ${ }^{4} \mathrm{He}$ isotope, ${ }^{3} \mathrm{He}$ has a nuclear spin of $1 / 2$ and obeys Fermi statistics. Its enthalpy $H$ therefore depends strongly on the density $x$ such that $H \sim T_{F}{ }^{-1}$ $\sim x^{-2 / 3}$ where $T_{F}$ is the Fermi temperature. At a given temperature, the dilution of $3 \mathrm{He}$ in a solution rich in $4 \mathrm{He}$ decreases the density and increases the enthalpy of the system. As the solubility of $3 \mathrm{He}$ is limited to $6.2 \%$ at low temperature, it is possible to work along the phase separation line which results in strong cooling by adiabatic dilution ( $T_{\text {final }} \approx$ $\left.0.3 T_{\text {initial }}\right)$. The use of heat exchangers to pre-cool the mixture allows the cryostat to operate between $2 \mathrm{mK}$ and $1 \mathrm{~K}$ with a cooling power ranging from $0.1 \mathrm{~mW}$ to a few $\mathrm{mW}$, depending upon temperature and circulation rate. These machines in turn opened the way to the demonstration of $3 \mathrm{He}$ superfluidity and solid ${ }^{3} \mathrm{He}$ antiferromagne-

Pierre Monceau has been the Director of the CRTBT, 25, av. des Martyrs, F-38042 Grenoble Cédex, since 1990. He joined the CNRS after receiving his doctorate from the University of Grenoble in 1970 for work on superconductors. A member of the CNRS national committee for solid-state physics since 1987 , he won the 1982 CNRS Médaille d'Argent.

\section{P. Monceau \\ Centre de Recherches sur les Très Basses Températures (CRTBT) CNRS, Grenoble, France}

tism [1], as well as the $\mu \mathrm{K}$ temperatures needed for studies of nuclear magnetism.

Dilution refrigerators are now frequently used in low-temperature laboratories to cool samples to $10 \mathrm{mK}$; they are also available commercially. Since the performance of 2 $\mathrm{mK}$ is now close to the theoretical limit, other techniques must be sought. Today's research thus consists mainly in adapting the basic techniques to different experimental conditions. The CRTBT, for example, aims to develop refrigerators for specific applications in space and at accelerators and underground particle detectors (see page 95). Another development, described by G. Frossati and N.F. de Oliveira on page 108 , aims to increase the ratio of the applied magnetic field $B$ to the temperature (i.e., $B / T$ ) by a factor of 20 .

\section{Bolometry}

Recent developments in bolometric selection have yielded spectacular improvements in energy resolution for various types of radiation; cooling to $10-100 \mathrm{mK}$ using a dilution cryostat is generally required. For measurements of particles or electromagnetic radiation, the most commonly used detector exploits a semiconductor to convert energy into electron-hole pairs. This allows direct measurements but presents two main disadvantages: the energy gap prevents the detection of low-energy particles and the energy dissipated in the detector originates from both the detection process (pair generation) and heating of the detector. Separating these two components is done using statistics so the energy resolution cannot be very high.

The problems are eliminated using a detector (called a bolometer) in which only heating takes place [2]. For detection, a very small change in the detector temperature must be correlated with a small change in energy. This is possible at very low temperatures where the specific heat of insulating materials decreases as $T^{3}$. For example, if one takes a small crystal of diamond of $2 \mathrm{~g}$, an incoming particle of $1 \mathrm{keV}$ increases $T$ by $10 \mathrm{mK}$ to $20 \mathrm{mK}$, thus yielding a resolution of a few eV. The main technical challenge is to ensure a low specific heat and good thermal contact for the couple between the thermometer and the detector. One can use semiconductor thermometers (mainly doped $\mathrm{Ge}$ or $\mathrm{Si}$ ) or superconducting devices (Josephson junctions or thin films near the critical temperature), all of which involve basic problems of solid-state physics.

High-sensitivity bolometers measuring very low fluxes are needed to improve observation techniques in astronomy. For instance, direct information on the composition and temperature of interstellar matter can be obtained using very high resolution measurements of $x$-rays. Sub-millimetre observations covering the range of the main emissions of cold matter in the Universe are also important for they allow a better understanding of the formation of the Universe after the Big Bang. However, experiments need to be placed aboard a balloon or satellite as the Earth's atmosphere absorbs radiation.

A second application concerns dark matter. The amount of matter in the Universe appears to be larger than the observed mass. The discrepancy may be due to particles with a finite mass but with a very small interaction with normal matter. To detect the particles, one needs a detector with a large mass shielded from all other radiation (e.g., buried deep underground to shield out cosmic radiation). If such a dark particle interacts with a nucleus in the detector, a small part of its energy is deposited (a few keV are expected). Bolometers able to measure this energy require a $10 \mathrm{~kg}$ crystal of $\mathrm{Ge}$ cooled to $10 \mathrm{mK}$.

\section{Quantum Fluids \\ Polarized ${ }^{3} \mathrm{He}$}

The microscopic description of $3 \mathrm{He}$ remains controversial. It is probably the simplest example of a Fermi liquid, i.e., a liquid made of interacting, degenerate fermions (other examples are electrons in metals and neutrons in nuclear matter). Understanding

CRTBT (in English, the Centre for Research at Very Low Temperatures) was established in Grenoble in the early1960 's. This relatively large laboratory belongs to, and was founded by, the French Centre National de la Recherche Scientifique (CNRS). The staff comprises about 65 scientists and engineers (twothirds being employed full time and the remainder having teaching duties), 40 qualified technicians and support staff, and about 10 visitors. Some 30 graduate students are usually preparing Ph.D. theses, slightly less than half of whom come from outside France. 
can be improved by studying the effect of polarizing the nuclear $1 / 2$ spins to make $3 \mathrm{He}$ atoms more alike, and to increase the in fluence of the Pauli principle, thereby modifying thermodynamic and transport properties. Several experiments have been performed during the last decade to determine these modifications, but a fundamental difficulty is to obtain significant nuclear polarization. Owing to the weakness of the ${ }^{3} \mathrm{He}$ nuclear moment combined with Fermi statistics, a magnetic field of $30 \mathrm{~T}$ (today's upper limit for static fields in the laboratory) polarizes the spins by only $10 \%$ ( $55 \%$ up spins, $45 \%$ down spins). However, larger polarizations are possible out of equilibrium due to the long magnetic relaxation time (several hundreds of seconds).

We are presently using two different methods based on this idea. In the first, an initial large polarization is obtained by melting a highly spin-polarized solid (typical polarization of $80 \%$ at $5 \mathrm{mK}$ in a $11 \mathrm{~T}$ field). It has recently allowed the magnetization curve of spin-polarized ${ }^{3} \mathrm{He}$ to be measured up to $200 \mathrm{~T}$ [3]. An original aspect of the experiment is that the effective field felt by the $3 \mathrm{He}$ atoms is deduced from the heat released during the relaxation process. The results (Fig. 1) show that the susceptibility decreases with increasing field, bringing into question the otherwise appealing description of liquid $3 \mathrm{He}$ as a "nearly-localized" solid. We aim to confirm this result by precision measurements in large static fields (in collaboration with Nijmegen University).

The second method is based on the difference in the molar susceptibilities of concentrated and dilute liquid $3 \mathrm{He}$. The difference makes it possible to separate the ${ }^{3} \mathrm{He}$ atoms with an up-spin from those with a down-spin using fractional distillation in a counterflow of concentrated and dilute ${ }^{3} \mathrm{He}$. The procedure is performed in a ${ }^{4} \mathrm{He}$ circulating dilu-

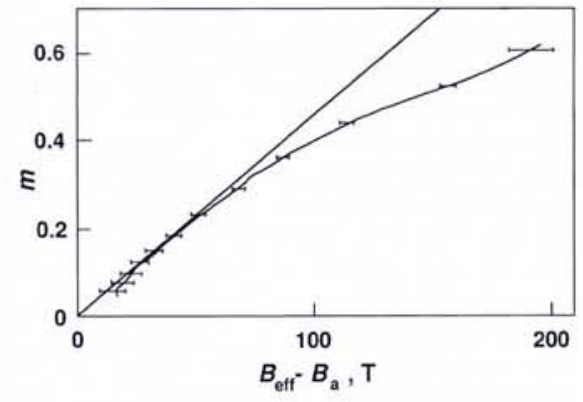

Fig. 1 - Polarization of liquid ${ }^{3} \mathrm{He}$ : the polarization is plotted as a function of the effective magnetic field at $70 \mathrm{mK}$ and 26 bars [3]. The initial polarization was obtained by melting a highly spin-polarized solid (typical polarization of $80 \%$ at $5 \mathrm{mK}$ in a $11 \mathrm{~T}$ field). The decreasing susceptibility on increasing the field brings into question the appealing description of liquid ${ }^{3} \mathrm{He}$ as a "nearly-localized" solid.

tion refrigerator, and a stationary polarization of $15 \%$ (seven times the equilibrium polarization in the $6.6 \mathrm{~T}$ field) at $14 \mathrm{mK}$ has been obtained.

\section{Dilute gas cooling}

Dilute gas cooling - a part, in fact, of atomic physics - has been actively studied by French groups, particularly this last decade. Gas atoms can be efficiently cooled by laser trapping and cooling (caesium vapour, for example, has been cooled to the $\mu \mathrm{K}$ range [6]). As a result, the hydrogen maser for a frequency standard has been improved considerably and more recently, a group at Harvard used a hydrogen maser to explore non-linearity in quantum mechanics.

A large international effort was devoted in the 1980's to Bose condensation in spinpolarized atomic hydrogen. An assembly of

\section{Refrigerators for Special Applications}

The CRTBT's development of refrigerators for specific applications has focussed on miniaturisation, high vacuum operation, flexibility, and reliability. The first was a dilution refrigerator with much simplified operation, achieved by eliminating the ${ }^{3} \mathrm{He}$ liquefaction stage using a heat exchanger to recuperate the enthalpy of the evaporating gas in the still. This resulted in a miniature device (now made under licence by the company TBT) of small diameter $(29 \mathrm{~mm})$ that can be rapidly cycled after sample changes; it is well adapted to magnetization measurements in high magnetic fields (17 T).

It is sometimes impractical to have a liquid helium dewar surrounding the refrigerator and then achieve rapid cooling by circulation. We have developed a dilution refrigerator using a patented, double boiler (still) technique: a separate cryostat contains the liquid $\mathrm{He}$ bath so the sample can be kept at $10 \mathrm{mK}$ to $300 \mathrm{~K}$, even under a high vacuum where heat transfer is limited.

A third approach combining these two techniques involves a cryostat without the conventional $1 \mathrm{~K}$ pot for the liquefaction of ${ }^{3} \mathrm{He}$ and a double boiler. It was persued in order to make an "upside-down" dilution refrigerator; samples are attached to the "top" of the refrigerator, which is then protected by copper radiation shields and sealed in a leak-tight cover. This new type of cryostat may be used in a standard ${ }^{4} \mathrm{He}$ storage vessel, with the actual refrigerator resting on top (see cover illustration). Flexibility and an unmatched ease of use makes it a very attractive alternative to the conventional dilution refrigerator.

Finally, a miniature dilution refrigerator unit that can function unattended on a satellite encompasses some difficult constraints. For this we have developed a novel type of cryostat based on the extraction of ${ }^{3} \mathrm{He}$ from the dilute phase by a high-speed flow of superfluid ${ }^{4} \mathrm{He}$ (CNRS patent). This type of refrigerator cannot recycle the used mixture (due to absence of a still). Fortunately, the liquid consumption is extremely small and the system can work automatically for up to 5 years with a reservoir of only a few litres; the absence of a pump ensures high reliability. A prototype has been tested and a cryostat is in the process of being made for experiments on a solar telescope for submillimetre radiation detection; it will demonstrate the technically feasibility of a space telescope with a detector cooled to below $100 \mathrm{mK}$. weakly interacting hydrogen atoms, polarized in a strong magnetic field, is a boson system since the atoms carry a total spin of zero or one (deuterium, on the other hand, obeys Fermi statistics). Due to its very large zero-point energy, spin-polarized hydrogen remains a gas all the way down to $0 \mathrm{~K}$ : it improves upon ${ }^{4} \mathrm{He}$ and $3 \mathrm{He}$ which remain liquid (at moderate pressures). This, then, provides us with a unique opportunity to investigate Bose-Einstein condensation (BEC) in an interacting system, where the well-documented interaction - of the raregas type - can be largely modulated by changing the gas density.

Two different types of experiments have been carried out in this very challenging area [4]. Using extreme conditions (10 T; $0.3 \mathrm{~K}$ ), very high densities (number of atoms per unit volume $n>10^{19}$ atoms $/ \mathrm{cm}^{3}$ ) have been reached. However, efforts aiming at $\mathrm{BEC}$ failed due to relaxation and very energetic recombination processes so the highdensity route is presently on stand-by Recalling that the Curie temperature $T_{c}$ varies as $n^{2 / 3}$, some groups turned to exploiting lower densities at $\mathrm{mK}$ temperatures or below. The evaporation of magnetically trapped atoms to cool hydrogen has been tested to a few $\mathrm{mK}$; microwave traps have also been proposed. The breakthrough to BEC might in the end come from an optical scheme presently under investigation where spin-polarized hydrogen is laser cooled in a magnetic field trap using Lyman $\alpha$ radiation.

\section{Adsorbed ${ }^{3} \mathrm{He}$}

It is well known from work on bulk samples that quantum tunnelling arises in solid ${ }^{3} \mathrm{He}$ owing to the large zero-point kinetic energy of the particles with their small masses. The multiple spin exchange Hamiltonian (the most natural extension of the Heisenberg model) provides a very good description of the three-dimensional magnetism of bulk $3 \mathrm{He}$ by introducing the coherent exchange of rings of several particles (see G. Frossati and N.F. de Oliveira, page 108). The exchange interaction between $3 \mathrm{He}$ atoms in two-dimensional systems should therefore lead to interesting effects. The "two-dimensional magnets" studied at the CRTBT consist of $3 \mathrm{He}$ layers of atomic thickness adsorbed at low temperatures on an exfoliated graphite substrate (surface area of $50 \mathrm{~m}^{2}$ ). The sample, at about $1 \mathrm{mK}$, is located in a cell attached to a dilution refrigerator or a nuclear demagnetization stage; magnetic properties are most conveniently determined using NMR.

The density of adsorbed atoms can be adjusted to produce 2-d systems in both liquid and solid phases, as well as in more exotic phases ("commensurate phases") due to the locking of the lattice parameter of the adsorbate to that of the substrate. Multilayers are limited in thickness to about 10 layers by capillary condensation in the substrate.

A detailed investigation of the magnetic properties of these systems has been performed for a large coverage range with emphasis on the solid-like systems [5]. A multilayer with about 2.5 atomic layers displays an extraordinary ferromagnetism; the 
magnetic behaviour is determined by the second layer, a solid of triangular structure and relatively large lattice parameter where quantum exchange is particularly important. The magnetic susceptibility of this particular system is well described by the exact, hightemperature series expansion for the 2-d ferromagnetic Heisenberg Hamiltonian on a triangular lattice. The expansion does not diverge at any finite temperature in the measuring range, even at temperatures as low as $\theta / 9$ (Fig. 2), where $\theta$ is the Curie-Weiss temperature. This is the first time that agreement has been found between theory and predictions. The suppression of the ordering temperature predicted by Mermin and Wagner owing to the reduced dimensionality was clearly demonstrated.

Several other systems are presently being investigated, and there is convincing evidence that new experimental model systems for fermions on a lattice will soon be discovered. The 2-d Heisenberg antiferromagnet on a triangular lattice, studied extensively in recent years, is certainly a good candidate.

\section{Complex Systems}

Very different fields of research sometimes raise similar problems. So it is for fluid turbulence and glassy and glass-like systems. This is why we choose to present these fields in a parallel, and maybe provocative, manner.

\section{Turbulence}

Turbulence may appear as an exotic problem for low-temperature physicists. However, it touches the heart of their traditional preoccupations, both technical and conceptual. For instance, gaseous ${ }^{4} \mathrm{He}$ is a very interesting fluid for studying strongly turbulent flows because its critical point at $5 \mathrm{~K}$ and 2 bars, lying near the regime where the kinematic viscosity is low, is particularly convenient. Reynolds numbers $R e$ at submetre scales can be increased spectacularly (using a jet one reaches $R e \approx 10^{6}$ with a nozzle of $2 \mathrm{~mm}$ in diameter) making an ordinary experimental vessel the equivalent of a wind tunnel. This, of course, calls for the evaporation of litres per minute of liquid ${ }^{4} \mathrm{He}$, and thus the handling $\mathrm{m}^{3}$ per minute of gas at room temperature - standard practice for low-temperature laboratories.

The well-established hot-wire technique for velocity measurements can be improved using low-temperature approaches (e.g., micron and $\mathrm{MHz}$ resolutions are possible with superconducting films on a glass fibres). On the conceptual side, turbulence raises fundamental problems in statistical physics, which is in turn traditionally linked to low-temperature research. For instance, we discuss below how the hierarchy of scales in turbulence could be similar to the hierarchy of time scales for relaxations in glasses and spin glasses.

\section{Disorder}

Disordered systems are undoubtedly encompassed by low-temperature physics owing to their unique properties. In the early 1970 's, it was observed that structural disorder found in a few elementary insulating glasses induces specific properties at very

Fig. 2-Adsorbed ${ }^{3} \mathrm{He}$ : the inverse (square points) of the nuclear magnetic susceptibility of the second layer of a ${ }^{3} \mathrm{He}$ film adsorbed on graphite plotted as a function of temperature (the circles correspond to the inverse susceptibility multiplied by 10). The total thickness of the film is 2.5 atomic layers. The dashed line represents the CurieWeiss limiting behaviour at elevated temperatures, and the solid lines the 10-terms high-temperature series expansion for the 2-d ferromagnetic Heisenberg Hamiltonian. The $\exp (\mathrm{J} / \mathrm{T})$ behaviour, with $J=2.1 \mathrm{mK}$, predicted by the model

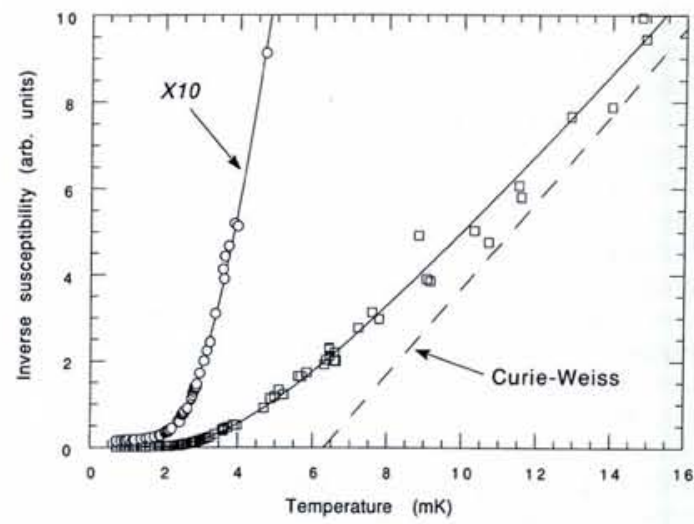
accurately describes the system, thus providing experimental support for the Mermin-Wagner theory that reduced dimensionality suppresses the ordering transition in this two-dimensional model magnet. From [5].

low temperatures (below $\approx 1 \mathrm{~K}$ ). For example, as discussed on page 105 by $P$. Esquinazi et al., in contrast to the phonon Debye behaviour obeyed by crystals, "anomalous" acoustic or thermal properties arise as a consequence of disorder. There are "defects" with a broad distribution of characteristic energy extending down to very low energies, the effects of which can be observed at very low temperatures.

Two new groups of materials that are also characterised by some kind of disorder, or frustration, are found to exhibit "glassy" properties at very low temperatures. There are the quasicrystals characterised by a quasiperiodic structure in three dimensions which can accommodate a local, icosahedral symmetry. AIMn quasicrystals, the first of the new class of material to have been synthesized, show magnetic properties which are totally different to those for their crystalline (periodic) counterparts. Surprisingly, they undergo a spin-glass transition owing to a fraction of the $\mathrm{Mn}$ atoms retaining the full magnetic moment despite the presence of the Al matrix. The origin of the moment is still a matter of debate and work is need to understand the quasicrystalline spin glasses. It is worth noting that the quasicrystalline spin-glass state is very similar to the one found in canonical crystalline $\mathrm{Cu}-\mathrm{Mn}$ or $\mathrm{Ag}-\mathrm{Mn}$, and that in $\mathrm{Al}-\mathrm{Mn}$ alloys, its characteristics closely resemble those of the amorphous state. Ö. Rapp describes on page

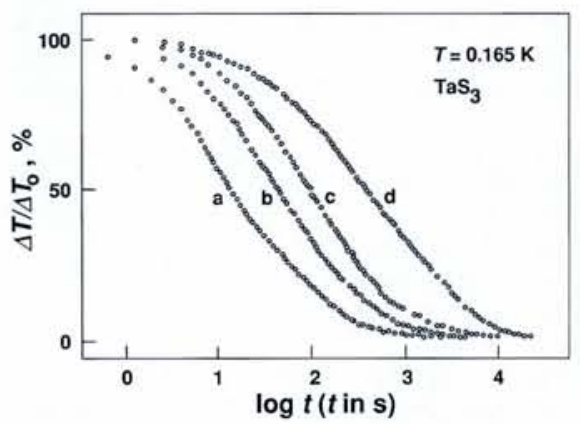

Fig. 3 - Charge-density wave (CDW) compound: aging effects in the quasi one-dimensional CDW compound $T_{a} S_{3} . \Delta T / \Delta T_{0}$ as a function of time for different waiting times $t=$ $0.5 \mathrm{~s}(\mathrm{a}), 20 \mathrm{~s}$ (b) $60 \mathrm{~s}(\mathrm{c}), 36 \mathrm{~h}(\mathrm{~d}) . \Delta T_{0}$ is the magnitude of the applied temperature incre. ment, switched off at time $t=0$. See [6].
104 quantum effects in transport properties of quasicrystalline $\mathrm{Al}_{63} \mathrm{Cu}_{25} \mathrm{Fe}_{12}$ which are, on the other hand, much larger than those found in amorphous metals.

The second group comprises quasi onedimensional metallic compounds in which the electronic charge condenses below the Peierls transition into a charge-density wave (CDW). In its ground state, the CDW is pinned to the ionic lattice through the interaction with impurities or lattice defects: this disordered character is at the origin of metastable states and of several "glassy" properties of the CDW. At very low temperatures, properties characteristic of disorder are revealed such as a contribution to the specific heat (in addition to the phonon contribution below $0.5 \mathrm{~K}$ ) with a smooth $T^{\alpha}$ temperature dependence (with $\alpha<1$ ) and of the same order of magnitude as in glasses. The result confirms the universality of this thermodynamic property. More spectacular is the non-exponential energy relaxation occurring after a thermal pulse, or a thermal perturbation of long duration, has been sent into the sample [6]. The kinetics of these long-time relaxations depend upon the time the perturbation was applied to the system - a phenomenon reminiscent of the relaxation of thermoremanent magnetization around the freezing temperature $T_{\mathrm{f}}$ in spin glasses, and of aging effects below $T_{f}$, i.e., effects of the time during which the system is maintained under external perturbation (magnetic field). In CDW materials, aging and a temperature decrease shift the spectrum of relaxation times of the metastable states towards very large values $\left(\approx 10^{5} \mathrm{~s}\right.$ or more at $100 \mathrm{mK}$; see Fig. 3)

\section{Possible connections}

There are possible and fruitful connections between disordered materials and turbulence which demonstrate the ubiquity of fundamental problems. In turbulent flows, the probability density functions (PDF) of velocity differences $V(x+r)-V(x)$ as a function of the position $x$ are strongly nonGaussian for small distances $r$. But the PDF can be fitted by combining a Gaussian with a distribution $\mathrm{G}_{r}(\sigma)$ of their variances $\sigma$. Similarly, the non-exponential energy relaxation in CDW materials can be fitted by combining exponential functions with a distribution $\mathrm{P}(\tau)$ of characteristic times $\tau$. The 


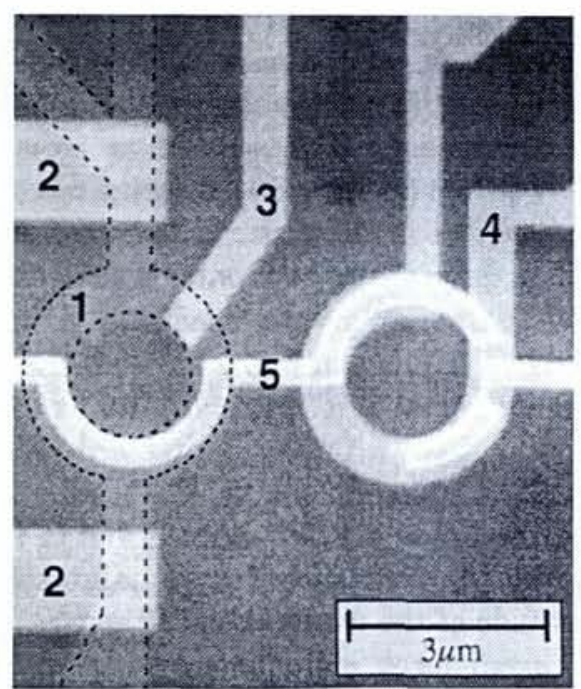

Fig. 4 - Mesoscopy: scanning electron micrograph [7] of a single metal ring with a SQUID magnetometer (numbers in parenthesis refer to numbers on the illustration). On the left, the ring (1) etched in GaAs in the GaAs-GaAlAs heterojunction (the dashed line has been added because of the poor contrast) with the two gates, (2) and (3). On the right, the calibration coil (4). To the top, the first level of the SQUID device (5) with the two microbridge junctions on the right (the picture was taken before fabricating the second level of the thin-film SQUID).

parallel is not artificial. In turbulent flows, the hierarchy of scales (where large-scale flow feeds energy to the smaller scales) is shown to yield for the distribution $\mathrm{G}_{r}$ a Gaussian in In $\sigma$. In the same way, hierarchical models for the relaxation in glassy materials (where small scales control the relaxation of larger ones) yield for the distribution $P(\tau)$ a Gaussian in $\ln \tau$. It is not the place here to develop the analysis; we simply want to illustrate the great unity of physics and the ubiquity of fundamental problems that can be addressed using low temperatures.

\section{Mesoscopy}

We and others have been particularly active over the past few years in the area of so-called mesoscopic physics. At adequately low temperatures (typically $1 \mathrm{~K}$ and below), coherence - and therefore interference effects - may develop in sufficiently clean, normal metal samples on the micrometre scale. As discussed on page 102 by Ö. Rapp, the phenomenon induces all kinds of new effects in transport measurements. For example, weak-localization, conductance fluctuations, and non-local effects may be observed in wires. A.G. Aronov, the winner of the 1993 Hewlett-Packard Europhysics Prize of the European Physical Society, describes on page 98 how the same physics, in the presence of magnetic fields, yields conductance oscillations with periodicity $h / e$ in a single ring (AharonovBohm oscillations) and $h / 2 e$ on averaging over a large number of rings (array of rings or cylinders).

\section{Equilibrium properties}

The most exciting phenomena involve the equilibrium properties of such systems. In a conducting ring where phase coherence is present, the quantum properties of the electronic wave-functions should be taken into account. The fundamental state is therefore sensitive to the fact that the flux inside the ring is not an integer multiple of the flux quantum $\phi_{\mathrm{o}}$. Under these conditions, theory predicts a current circulating around the ring without any dissipation. This phenomena is completely different from the current circulating in a superconductor ring because, in the normal state, the resistance of the ring is finite.

Measuring this very small current in the $\mu \mathrm{m}$-sized ring is extremely difficult. The magnetization created by the current is equivalent to the one created by 1000 magnetic spins, and represents a very small energy.

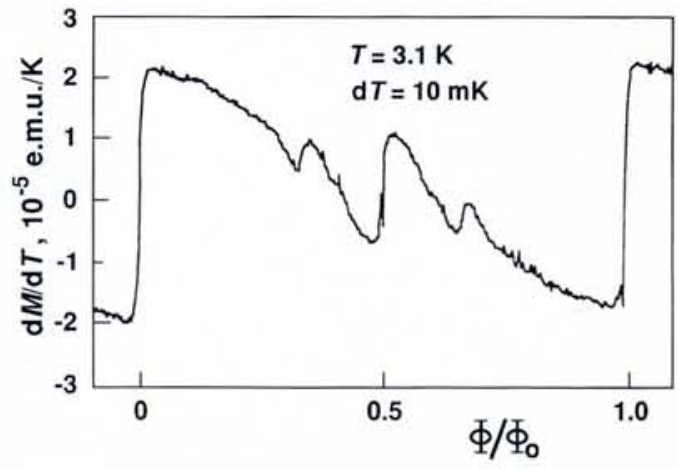

Fig. 5 - Superconducting arrays: the rate of change with temperature of the magnetization $M$ of a square network of superconducting In wires, plotted as a function of the normalised flux per network plaquette, shows magnetization jumps at rational and integer frustration $(3.12 \mathrm{~K} ; 0.45 \mu \mathrm{m}$ wide, $0.1 \mu \mathrm{m}$ thick filaments; $3.2 \mu \mathrm{m}$ periodicity). From [8]. In collaboration with the L2M laboratory at Bagneux, France, we have developed a circuit comprising a ring of $3 \mu \mathrm{m}$ diameter, made in a GaAs-AIGaAs heterojunction, with a direct-current SQUID of the same dimensions realised by electron lithography (Fig. 4). The device allows a very good coupling between the magnetization and current measurements. Results [7] show good agreement with theoretical predictions, in contrast to recent experiments at IBM and Bell Labs which gave a signal two orders of magnitude larger. New experiments with different samples are in preparation to understand the origins of the important discrepancy.

The observed phenomena are not only reminiscent of, but complementary at a deep level to, the well-known AharonovBohm flux quantization and localization effects. These have also been studied in detail using both normally conducting and superconducting artificial arrays of submicron size obtained by microfabrication techniques [8]. Resistance and magnetization measurements (made possible by the development at the CRTBT of specialized electronic instrumentation for low temperatures) show that the transition between normal conductivity and superconductivity does not vary monotonically owing to frustration effects induced by an applied magnetic field (Fig. 5).

\section{Single-electron tunnelling}

A spectacular manifestation of quantum effects at very low temperatures is singleelectron tunnelling which has been observed recently in small tunnel junctions [9]. These fundamental experiments involve the controlled transfer of individual charges in semiconducting, metallic or superconducting islands through a series of tunnel barriers having capacitance below $10^{-15}$ farads. The tunnelling channel, normally closed at $\mathrm{mK}$ temperatures when the Coulomb energy exceeds the thermal energy (Coulomb blockade), is opened in a controlled way by a small electrical bias on a gate electrode. This experiment bridges the current versus frequency gap in the famous metrology triangle, opening the principle of an independent measurement of the electronic charge. The other sides of the triangle are frequency/voltage and voltage/current, corresponding to other famous low-temperature effects (the Josephson and quantum Hall effects, respectively).

\section{Future Developments}

Low temperatures are, by necessity one might say, a very active field in fundamental research as well as in technology and instrumentation. Progress naturally requires an important theoretical effort. Numerical simulations are developing very rapidly, especially in the context of high-temperature ceramic superconductors (e.g., strongly-correlated fermions, 2-d Hubbard model, magnetic environment around vacancies). More generally, it seems that we are presently at a crossroads between the study of frustration and disordered systems per se and progress in quantum coherence and quantum dynamics in these systems. This trend is, in itself, characteristic of progress in low-temperature physics and techniques.

\section{ACKNOWLEDGEMENT}

I am indebted to A. Benoit, J.L. Bret, B. Castaing, B. Hébral, J.C. Lasjaunias, B Pannetier, M. Papoular, and P.E. Wolf for their contributions to this article, and especially to M. Papoular for his constant help.

[1] Thuneberg E.V. \& Pekola J.P., Europhys. News 22 (1991) 3.

[2] Fiorini E., Europhys. News 23 (1992) 207. Phys. Rev. Lett. 66 (1991) 2895.

[4] J. Opt. Soc. of Amer. B 6 (1989); Spin Polarized Quantum Systems, ISI, Ed.: S. Stringari (World Scientific,1989).

[5] Godfrin H., Rapp R.E. \& Lauter H.J., Physica A 163 (1990) 101.

[6] Biljakovic K., et al., Phys. Rev. Lett. 67 (1991) 1902.

[7] Mailly D., Chapelier C. \& Benoit A., Phys. Rev. Lett. 70 (1993) 2020

[8] Pannetier B., in Quantum Coherence in Mesoscopic Systems B 254 (1991) 457, Ed.: B. Kramer (Plenum); Gandit P., et al., Europhys. Lett. 3 (1987) 623; idem, Physica B 152 (1988) 32

[9] Single Charge Tunneling, Eds.: M.H. Devoret \& H. Grabert, Proc. NATO ASI, Les Houches (1991).
[3] Wiegers S.A.J., Wolf P.E. \& Puech L. 